\title{
POSITIVE MAPS ON SELF-DUAL CONES
}

\author{
B. IOCHUM
}

(Communicated by Paul S. Muhly)

\begin{abstract}
Different properties of maps on a self-dual cone which preserve the order and the orthogonal decomposition are studied: polar decomposition, link with the order isomorphisms, normality, extremality within the order preserving maps. A characterization of derivation preserving maps is given. The results generalize a previous approach by S. Yamamuro et al.
\end{abstract}

\section{INTRODUCTION AND NOTATION}

In a series of papers, S. Yamamuro has emphasized the interest of orthogonally decomposable spaces $[12,13,14]$. A nice example is given by a Hilbert space $H$ ordered by a self-dual cone $H^{+}$: each vector in $H$ can be uniquely decomposed in orthogonal elements of $\mathrm{H}^{+}$. So it is interesting to characterize the maps which preserve the order and also the orthogonal decomposition.

In 1974, A. Connes showed that there is a one-to-one correspondence between $\sigma$-finite von Neumann algebras and orientable, facially homogeneous self-dual cones in complex Hilbert spaces [3]. It is in this setting that S. Yamamuro and his collaborators $[4,5,7,12-18]$ have studied the orthogonal decomposition (o.d.) homomorphisms of the natural cone $\mathfrak{P}_{M, \xi_{0}}$ associated to a von Neumann algebra $M$ with a cyclic and separating vector $\xi_{0} \in H$ (see the review [19] and the Ph.D. thesis of T. Dang). Their main tool is the deep Tomita-Takesaki theory.

Here we generalize most of their results assuming only that $\mathrm{H}^{+}$is a facially homogeneous self-dual cone. It has been shown in [8] that there exists a oneto-one correspondence between these cones and the Jordan Banach algebras. However we do not use the algebraic part of this correspondence. Thus the interest of the results lies not only in the generalization but also in the fact that the only used tool is the order. In particular this direct approach avoids the Tomita-Takesaki theory and works in the real vector space case which in general is more complicated than the complex one.

Received by the editors September 18, 1989.

1980 Mathematics Subject Classification (1985 Revision). Primary 46L10, 17C65.

Key words and phrases. Positive map, self-dual cone, orthogonal decomposition. 
In the following, $H$ is a real Hilbert space, $H^{+}$is a self-dual cone in $H$ which means that

$$
H^{+}=\left\{\xi \in H \mid\langle\xi, \eta\rangle \geq 0 \forall \eta \in H^{+}\right\} .
$$

If $\xi \in H$ then $\xi=\xi^{+}-\xi^{-}$where $\xi^{ \pm} \in H^{+}$and $\left\langle\xi^{+}, \xi^{-}\right\rangle=0$. This Jordan decomposition is unique so the absolute value $|\xi|=\xi^{+}+\xi^{-}$is well defined.

If $F$ is a face, $F^{\perp}=\left\{\xi \in H^{+} \mid\langle\xi, \eta\rangle=0 \forall \eta \in F\right\}$ is a face called the orthogonal face. When $\xi \in H^{+},\langle\xi\rangle$ denotes the face generated by $\xi$. $\mathscr{F}\left(H^{+}\right)$ is the lattice of complete faces (i.e. $F=F^{\perp \perp}$ ).

$\xi \in H^{+}$is called a quasi-interior point if $\langle\xi\rangle^{\perp}=\{0\}$.

Let $L(H)$ be the set of bounded operators on $H, L\left(H^{+}\right)$be the subset of order preserving operators, and $D\left(H^{+}\right)=\left\{\delta \in L(H) \mid e^{t \delta} \in L\left(H^{+}\right) \forall t \in \mathbf{R}\right\}$ be the set of derivations. Recall that

$\delta \in D\left(H^{+}\right)$if and only if $\langle\delta \xi, \eta\rangle=0$

$$
\text { when }\langle\xi, \eta\rangle=0 \text { and } \xi, \eta \in H^{+}[8, I .2 .3] \text {. }
$$

The order ideal of $H$ is

$$
Z_{H^{+}}=\left\{T \in L(H) \mid \exists \alpha_{T} \in \mathbf{R}^{+},-\alpha_{T} \xi \leq T \xi \leq \alpha_{T} \xi \forall \xi \in H^{+}\right\} .
$$

Let $U\left(H^{+}\right)$be the unitaries in $L\left(H^{+}\right)$and

$$
S\left(H^{+}\right)=\left\{U \in U\left(H^{+}\right) \mid[U, T]=0 \forall T \in Z_{H^{+}}\right\}
$$

be the set of symmetries.

$H^{+}$is said to be indecomposable if $Z_{H^{+}} \approx \mathbf{R}$.

$\xi \in H^{+}$is called a trace vector when $S \xi=\xi \forall S \in S\left(H^{+}\right)$.

If $N_{F}=P_{F}-P_{F^{\perp}}$ is in $D\left(H^{+}\right)$then $H^{+}$is said to be facially homogeneous. Here $P_{F}$ denotes the orthogonal projection on the closure of the vector space generated by $F$. When $H^{+}$is facially homogeneous, the self-adjoint part $\mathscr{M}$ of $D\left(H^{+}\right)$has a natural structure of J. B.-algebra [8, III.2.1] such that its center coincides with $Z_{H^{+}}$[8, III.3.6].

$G L\left(H^{+}\right)$denotes the order isomorphisms $A$ of $H^{+}$(i.e. $A, A^{-1} \in L\left(H^{+}\right)$) . This paper is mainly concerned with the study of the set $O D\left(\mathrm{H}^{+}\right)$of orthogonal decomposition (o.d.) homomorphisms defined by

$$
\left\{A \in L\left(H^{+}\right) \mid\langle A \xi, A \eta\rangle=0 \text { if } \xi, \eta \text { are orthogonal in } H^{+}\right\} \text {. }
$$

$G L O D\left(H^{+}\right)$denotes the o.d. isomorphisms $A$ (i.e. $A, A^{-1} \in O D\left(H^{+}\right)$) . If $A \subset L(H)$ then $(A)^{+}$means the set of positive operators in $A$.

After different characterizations of o.d. homomorphisms (Proposition 2) we study the derivation preserving maps because when $A \in O D\left(H^{+}\right)$, the map

$$
\alpha_{A}: B \in L(H) \rightarrow A^{*} B A \in L(H)
$$

preserves $D\left(H^{+}\right)$(Theorem 4). The polar decomposition of $A \in O D\left(H^{+}\right)$ is within $O D\left(H^{+}\right)$. Thus it is possible to consider the links between the 
order isomorphisms and the o.d. isomorphisms. A bijective o.d. homomorphism is an o.d. isomorphism (Lemma 7). Only Banach lattices are such that $G L\left(H^{+}\right) \subset G L O D\left(H^{+}\right)$(Proposition 11). The normality of o.d. homomorphisms is then considered and in particular we pay attention to the cones $H^{+}$ such that any element in $O D\left(H^{+}\right)$is normal. This property is characterized in Proposition 14 for the finite-dimensional case. Some results on semi-groups of o.d. homomorphisms are also considered but since they are obvious extensions of known results, the proofs are left to the reader. Finally it appears that o.d. homomorphisms are extreme vectors in $L\left(H^{+}\right)$(Lemma 17), and we characterize the cones $H^{+}$such that the extreme points in $L\left(H^{+}\right)$are o.d. homomorphisms (Theorem 18).

\section{ChARACTERIZATIONS OF o.d. HOMOMORPHISMS}

Lemma 1. Let $H^{+}$be a self-dual cone. Then $\mathscr{M} \cap L\left(H^{+}\right) \subset\left(Z_{H^{+}}\right)^{+}$.

Proof. Let $\delta \in \mathscr{M} \cap L\left(H^{+}\right)$. We may assume that $\delta$ is positive as operator. Then for $\xi, \eta$ orthogonal in $H^{+}$, we claim that $\left\langle\delta^{n} \xi, \eta\right\rangle=0$ thus $\delta^{n} \in \mathscr{M}$ for any integer $n$. In fact, $\left\langle\delta^{2} \xi, \eta\right\rangle=\langle\delta(\delta \xi), \eta\rangle=0$ because $\langle\delta \xi, \eta\rangle=0$ and $\delta$ preserves the order. The claim is proved by recurrence on $n$. Hence all polynomials in $\delta$ are in $\mathscr{M}$. By spectral theory, the spectral projections of $\delta$ are also in $\mathscr{M}$, so by [8, I.2.6], $\delta$ is in $Z_{H^{+}}$. Moreover, if $\xi \in H$, $\langle\delta \xi, \xi\rangle=\left\langle\delta \xi^{+}, \xi^{+}\right\rangle+\left\langle\delta \xi^{-}, \xi^{-}\right\rangle \geq 0$ so $\delta$ is positive.

The following is an extension of $[5,2.1]$ and $[17,2.2]$.

Proposition 2. Let $H^{+}$be a self-dual cone and $A \in L(H)$. The following conditions are equivalent:

(i) $A \in O D\left(H^{+}\right)$,

(ii) $A \in L\left(H^{+}\right)$and $|A| \in Z_{H^{+}}$,

(iii) $|A \xi|=A|\xi| \quad \forall \xi \in H$.

In particular $\left(Z_{H^{+}}\right)^{+} \subset O D\left(H^{+}\right)$.

Proof. The implications (i) $\Leftrightarrow$ (ii) are an immediate consequence of Lemma 1 and of the fact that $Z_{H^{+}}$is the self-adjoint part of an abelian von Neumann algebra [8, I.2.7].

(i) $\Rightarrow$ (iii): Let $A \in O D\left(H^{+}\right)$and $\xi=\xi^{+}-\xi^{-} \in H$.

Since $A \xi^{ \pm} \in H^{+}$and $\left\langle A \xi^{+}, A \xi^{-}\right\rangle=0,(A \xi)^{ \pm}=A \xi^{ \pm}$by the uniqueness of the Jordan decomposition of $A \xi[8$, I.1.2]. Thus $|A \xi|=A|\xi|$.

(iii) $\Rightarrow$ (i): Assume $|A \xi|=A|\xi| \quad \forall \xi \in H$. Obviously $A \in L\left(H^{+}\right)$. Let $\xi, \eta$ be two orthogonal vectors in $H^{+}$. Then

$$
\begin{aligned}
\langle A(\xi-\eta), A(\xi-\eta)\rangle & =\langle|A(\xi-\eta)|,|A(\xi-\eta)|\rangle \quad \text { by definition of }|.| \\
& =\langle A|\xi-\eta|, A|\xi-\eta|\rangle \\
& =\langle A(\xi+\eta), A(\xi+\eta)\rangle
\end{aligned}
$$

and $\langle A \xi, A \eta\rangle=0$. 
If $A \in\left(Z_{H^{+}}\right)^{+}$then by spectral theory, all its spectral projections are in $Z_{H^{+}}$ $[8, \mathrm{I} .2 .7]$ and $A \in L\left(H^{+}\right)[8, \mathrm{I} .2 .6]$. Hence $A$ is in $O D\left(H^{+}\right)$.

\section{DERIVATION PRESERVING MAPS}

The previous proposition characterizes the elements of $O D\left(H^{+}\right)$among those in $L\left(H^{+}\right)$. Here is another characterization:

Lemma 3 (see [17, 2.2]). Let $H^{+}$be a self-dual cone and $A \in L(H)$. The following conditions are equivalent:

(i) $A \in O D\left(H^{+}\right)$,

(ii) $A \in L\left(H^{+}\right)$and $\alpha_{A}: B \in L(H) \rightarrow A^{*} B A$ maps $D\left(H^{+}\right)$into $D\left(H^{+}\right)$,

(iii) $A \in L\left(H^{+}\right)$and $\alpha_{A}\left(Z_{H^{+}}\right) \subset Z_{H^{+}}$.

Proof. Let $A \in O D\left(H^{+}\right)$and $\delta \in D\left(H^{+}\right)$. For $\xi, \eta \in H^{+}$satisfying $\langle\xi, \eta\rangle$ $=0$ we have $\langle A \xi, A \eta\rangle=0=\langle\delta A \xi, A \eta\rangle=\left\langle A^{*} \delta A \xi, \eta\right\rangle$ and $A^{*} \delta A \in D\left(H^{+}\right)$ [8, I.2.3]. If $\delta \in Z_{H^{+}}$is a positive operator then $\delta^{1 / 2} \in L\left(H^{+}\right)$[8, I.2.6, I.2.7] and $\delta^{1 / 2} A \in L\left(H^{+}\right),\left\langle\delta^{1 / 2} A \xi, \delta^{1 / 2} A \eta\right\rangle=0$. Thus $\delta^{1 / 2} A \in O D\left(H^{+}\right)$so $A^{*} \delta A=\left|\delta^{1 / 2} A\right|^{2} \in Z_{H^{+}}$by Proposition 2. Conversely if $\alpha_{A}$ maps $D\left(H^{+}\right)$in $D\left(H^{+}\right)$for $A \in L\left(H^{+}\right), A^{*} A=\alpha_{A}(\mathbf{1}) \in L\left(H^{+}\right)$is in $Z_{H^{+}}$by Lemma 1. Hence $A \in O D\left(H^{+}\right)$.

More generally it is possible to characterize the operator $A \in L(H)$ giving a derivation preserving map $\alpha_{A}$.

Theorem 4 (see $[17,1.3])$. Let $H^{+}$be a facially homogeneous self-dual cone and $\xi, \eta \in H$. The following conditions are equivalent:

(i) $\langle|\xi|,|\eta|\rangle=0$,

(ii) $\langle\delta \xi, \eta\rangle=0 \forall \delta \in D\left(H^{+}\right)$.

Corollary 5 (see [17,2.1]). Let $A \in L(H)$. The following conditions are equivalent

(i) $\alpha_{A}$ is derivation preserving,

(ii) $\langle|A \xi|,|A \eta|\rangle=0$ if $\langle|\xi|,|\eta|\rangle=0$.

Proof. (i) $\Rightarrow$ (ii): Let $\xi, \eta \in H$ and $\langle|\xi|,|\eta|\rangle=0$. By Theorem 4,

$$
0=\left\langle A^{*} \delta A \xi, \eta\right\rangle=\langle\delta A \xi, A \eta\rangle \quad \forall \delta \in D\left(H^{+}\right) .
$$

The same theorem gives $\langle|A \xi|,|A \eta|\rangle=0$.

(ii) $\Rightarrow$ (i): If $\xi, \eta \in H^{+}$are orthogonal, $\langle|A \xi|,|A \eta|\rangle=0$ by assumption.

Thus $(A \xi)^{ \pm}$is orthogonal to $(A \eta)^{ \pm}$; for instance

$$
0 \leq\left\langle(A \xi)^{+},(A \eta)^{+}\right\rangle \leq\langle|A \xi|,|A \eta|\rangle=0 .
$$

So if $\delta \in D\left(H^{+}\right), 0=\langle\delta A \xi, A \eta\rangle=\left\langle A^{*} \delta A \xi, \eta\right\rangle$ and $A^{*} \delta A \in D\left(H^{+}\right)$by [8, I.2.3].

Proof of Theorem 4. (i) $\Rightarrow$ (ii): $\langle|\xi|,|\eta|\rangle=0$ implies $\left\langle\xi^{ \pm}, \eta^{ \pm}\right\rangle=0$ by selfduality of $H^{+}$. Thus $\langle\delta \xi, \eta\rangle=0 \quad \forall \delta \in D\left(H^{+}\right)$. 
(ii) $\Rightarrow$ (i): Assume $\langle\delta \xi, \eta\rangle=0$ for all $\delta$ in $D\left(H^{+}\right)$. Let $F=\left\langle\xi^{+}\right\rangle, G=\left\langle\eta^{+}\right\rangle$ and $N_{F}=P_{F}-P_{F^{\perp}}, N_{G}=P_{G}-P_{G^{\perp}}$. We note that $\xi^{-} \in F^{\perp}$ thus $N_{F} \xi=|\xi|$ and similarly $N_{G} \eta=|\eta|$.

$$
\begin{aligned}
\langle|\xi|,|\eta|\rangle & =\left\langle N_{F} \xi, N_{G} \eta\right\rangle=\left\langle\left[N_{G}, N_{F}\right] \xi, \eta\right\rangle+\left\langle N_{F} N_{G} \xi, \eta\right\rangle \\
& =\left\langle N_{F} N_{G} \xi, \eta\right\rangle \text { because } N_{F}, N_{G} \text { are in the Lie algebra } D\left(H^{+}\right)
\end{aligned}
$$

$[8$, II.2.3]

$$
=\left\langle P_{F} N_{G} \xi^{+}, \eta\right\rangle+\left\langle P_{F^{\perp}} N_{G} \xi^{-}, \eta\right\rangle \quad \text { because } P_{F} N_{G} P_{F^{\perp}}=0
$$

$[8, \mathrm{I} .2 .4]$.

On the other hand, by hypothesis

$$
\begin{aligned}
0 & =\left\langle\mathfrak{P}_{F}\left(N_{G}\right) \xi, \eta\right\rangle \quad \text { using [8, III.2.3 i] and notations of [8, III.1.1]. } \\
& =\left\langle\mathfrak{P}_{F}\left(N_{G}\right)\left(P_{F} \xi^{+}-P_{F^{\perp}} \xi^{-}\right), \eta\right\rangle \\
& =\left\langle P_{F} N_{G} \xi^{+}, \eta\right\rangle \quad \text { by }[8, \text { III.1.1]. }
\end{aligned}
$$

Similarly $0=\left\langle\mathfrak{P}_{F^{\perp}}\left(N_{G}\right) \xi, \eta\right\rangle=-\left\langle P_{F^{\perp}} N_{G} \xi^{-}, \eta\right\rangle$. Thus $\langle|\xi|,|\eta|\rangle=0$.

\section{POLAR DECOMPOSITION}

When $H^{+}$is facially homogeneous it is known that if $A \in G L\left(H^{+}\right)$, its polar decomposition $U|A|$ satisfies $U \in U\left(H^{+}\right)$and $|A|=e^{\delta}$ where $\delta \in \mathscr{M}$ [8, II.3.2]. This is no longer true if $A \in L\left(H^{+}\right)$. Nevertheless the situation is better for o.d. homomorphisms:

Lemma 6 (see [6]). Let $H^{+}$be a self-dual cone and $A \in O D\left(H^{+}\right)$. If $U|A|$ is its polar decomposition, then $U$ and $|A|$ are in $O D\left(H^{+}\right)$.

Proof. By Proposition 2, $|A| \in\left(Z_{H^{+}}\right)^{+} \subset O D\left(H^{+}\right)$. Moreover $U$ is the strong limit of $A\left(n^{-1} \mathbf{1}+|A|\right)^{-1}$ when $n \rightarrow \infty$. Thus $U \in O D\left(H^{+}\right)$because $\left(n^{-1} \mathbf{1}+|A|\right)^{-1}$ is in $\left(Z_{H^{+}}\right)^{+} \subset O D\left(H^{+}\right)$(Proposition 2) and $O D\left(H^{+}\right)$is stable by product.

\section{ORDER ISOMORPHISMS AND o.d. ISOMORPHISMS}

In this paragraph we have gathered some results linking $G L\left(H^{+}\right)$and $G L O D\left(H^{+}\right)$.

Lemma 7 (see $[5,3.1 ; 1,2.1]$ ). Let $H^{+}$be a self-dual cone. If $A \in O D\left(H^{+}\right)$ and $A$ is bijective then $A \in G L\left(H^{+}\right)$. Moreover if $H^{+}$is facially homogeneous then $A \in G L O D\left(H^{+}\right)$.

Proof. Let $\xi \in H^{+}$and $\eta=A^{-1} \xi$. Then

$$
0 \leq\left\langle A \eta^{-}, \xi\right\rangle=\left\langle A \eta^{-}, A \eta^{+}-A \eta^{-}\right\rangle=-\left\|A \eta^{-}\right\|^{2} \leq 0
$$

and $\eta^{-}=0$. Thus $A^{-1} \in L\left(H^{+}\right)$. 
Assume $H^{+}$is facially homogeneous. As noticed before, $A=U e^{\delta}$ where $U \in U\left(H^{+}\right)$and $\delta \in \mathscr{M}$. Since $e^{2 \delta}=A^{*} A \in Z_{H^{+}},\left(A^{-1 *} A^{-1}\right)^{-1}=A A^{*}=$ $U e^{2 \delta} U^{*} \in Z_{H^{+}}\left[8\right.$, I.2.7] and $A^{-1} \in O D\left(H^{+}\right)$by Proposition 2.

Proposition 8 (see $[13,3.4]$ ). Let $H^{+}$be a self-dual cone with a quasi-interior point $\xi$. The following conditions are equivalent:

(i) $H$ is a Banach lattice,

(ii) $\mathscr{M} \xi$ is dense in $H$ and $G L\left(H^{+}\right) \subset G L O D\left(H^{+}\right)$.

Proof. (i) $\Rightarrow$ (ii): It is known that all order isomorphisms are o.d. isomorphisms in a Banach lattice. By [8, I.3.2], $Z_{H^{+}}=\mathscr{M}$ thus $\mathscr{M} \xi$ is dense in $H$.

(ii) $\Rightarrow$ (i): Let $\delta \in \mathscr{M}$. Then $e^{\delta} \in G L\left(H^{+}\right)$and $e^{2 \delta} \in Z_{H^{+}}$by hypothesis and Proposition 2. Hence $\delta \in Z_{H^{+}}$and $Z_{H^{+}}=\mathscr{M}$ since $Z_{H^{+}}$is included in $\mathscr{M}$ [8, I.2.7]. The proof of [8, I.3.2] gives the result.

Remark 9. Assume $\mathrm{H}^{+}$is facially homogeneous. Then

The condition $\mathscr{M} \xi$ dense in $H$ is automatically fulfilled [8, I.1.22].

Conditions (i) or (ii) are equivalent to $P(\delta) \in O D\left(H^{+}\right)$for all $\delta$ in $\mathscr{M}^{+}$ where $P(\delta)=2 \delta^{2}-\delta \circ \delta$ and $\circ$ is the Jordan product on $\mathscr{M}$ [8, III.4]. In fact for a face $F, P_{F}=P\left(2^{-1}\left(1+P_{F}-P_{F^{\perp}}\right)\right)$ [8, III.4.3] is in $O D\left(H^{+}\right)$ and $P_{F} \in Z_{H^{+}}$. Thus $H$ is a lattice by [8, I.3.2]. The converse follows from $\left(Z_{H^{+}}\right)^{+} \subset O D\left(H^{+}\right)$(Proposition 2).

As the following lemma shows, it is possible to find in general order homomorphisms preserving a chosen quasi-interior point in $\mathrm{H}^{+}$which are not o.d. homomorphisms.

Lemma 10 (see [18]). Let $H^{+}$be a self-dual cone with a quasi-interior point $\xi$. The following conditions are equivalent:

(i) $A \in L\left(H^{+}\right)$and $A \xi=\xi$ implies $A \in O D\left(H^{+}\right)$,

(ii) $A \in L\left(H^{+}\right), A$ bijective and $A \xi=\xi$ implies $A \in G L\left(H^{+}\right)$,

(iii) $H$ is isomorphic to $\mathbf{R}$.

Proof. The implication (i) $\Rightarrow$ (ii) is a consequence of Lemma 7.

(ii) $\Rightarrow$ (iii): The map $A: \eta \in H \rightarrow 2^{-1}\left(\eta+\|\xi\|^{-2}\langle\xi, \eta\rangle \xi\right)$ is clearly bijective and preserves the order and $\xi$. By assumption $A^{-1} \in L\left(H^{+}\right)$. Since

$$
A^{-1} \eta=2 \eta-\|\xi\|^{-2}\langle\xi, \eta\rangle \xi
$$

we have

$$
0 \leq\left\langle A^{-1} \eta^{+}, \eta^{-}\right\rangle=-\|\xi\|^{-2}\left\langle\xi, \eta^{+}\right\rangle\left\langle\xi, \eta^{-}\right\rangle \leq 0 .
$$

$\xi$ being quasi-interior, either $\eta^{+}=0$ or $\eta^{-}=0$. Hence, $H$ is totally ordered by $H^{+}$. Clearly $H^{+} \approx \mathbf{R}^{+}$.

With a stronger assumption; namely, $A \in G L\left(H^{+}\right)$and $\xi$ be a trace vector, $H$ is not necessarily reduced to $\mathbf{R}$. 
Proposition 11 (see [18]). Let $H^{+}$be a facially homogeneous self-dual cone with a quasi-interior trace vector $\xi$. If $A \in G L\left(H^{+}\right)$and $A \xi=\xi$ then $A$ is in $\operatorname{GLOD}\left(\mathrm{H}^{+}\right)$.

Proof. The extreme points of the order interval $[0, \xi]$ are of the form $P_{F} \xi$ for a face $F \in \mathscr{F}\left(H^{+}\right)\left[8\right.$, IV.2.3]. Let $A \in G L\left(H^{+}\right)$and $A \xi=\xi . A$ maps extreme points into extreme points of $[0, \xi]$. So if $F \in \mathscr{F}\left(H^{+}\right)$there exists $K, G \in \mathscr{F}\left(H^{+}\right)$such that $A P_{F} \xi=P_{G} \xi$ and $A P_{F^{\perp}} \xi=P_{K} \xi$. Since

$$
\begin{aligned}
P_{G} \xi+P_{G^{\perp}} \xi & =\xi \quad[8, \text { IV.2.1] } \\
& =A \xi=A\left(P_{F}+P_{F^{\perp}}\right) \xi=P_{G} \xi+P_{K} \xi,
\end{aligned}
$$

we get $\left\langle A P_{F} \xi, A P_{F^{\perp}} \xi\right\rangle=0$. This implies $\left\langle P_{F^{\perp}} A^{*} A P_{F} \eta, \rho\right\rangle=0$ for $\eta, \rho$ in $\langle\xi\rangle$ and $P_{F^{\perp}} A^{*} A P_{F}=0$ because $P_{\langle\xi\rangle}=P_{\langle\xi\rangle}{ }^{\perp \perp}=1$ [8, II.1.3].

Thus $A^{*} A \in \mathscr{M} \cap L\left(H^{+}\right) \subset Z_{H^{+}}$by [8, I.2.3] and Lemma 1, $A \in G L O D\left(H^{+}\right)$ by Proposition 2 and Lemma 7.

\section{NORMALITY IN $O D\left(H^{+}\right)$}

In this paragraph we study the cones with sufficiently many normal elements in $O D\left(H^{+}\right)$. Recall that $A \in L(H)$ is normal if $A^{*} A=A A^{*}$, quasinormal if $A$ and $A^{*} A$ commute and paranormal if $A^{* 2} A^{2}-2 \lambda A^{*} A+\lambda^{2} \geq 0 \forall \lambda \in \mathbf{R}^{+}$.

We remark first that if $H^{+}$is an indecomposable self-dual cone then $A \in$ $G L O D\left(H^{+}\right)$if $A=\lambda U$ with $\lambda \in \mathbf{R}^{+}, U \in U\left(H^{+}\right)$and every $A$ in $O D\left(H^{+}\right)$ is quasinormal.

Proposition 12 (see $[5,3.4 ; 16,1.2]$ ). Let $H^{+}$be a facially homogeneous selfdual cone.

(i) The following conditions are equivalent:

(a) Any o.d. isomorphism is normal,

(b) $U\left(H^{+}\right)=S\left(H^{+}\right)$.

(ii) If any o.d. homomorphism is paranormal then all o.d. isomorphisms are normal.

Proof. (i) (a) $\Rightarrow$ (b): Let $U \in U\left(H^{+}\right)$and $V \in Z_{H^{+}}$such that $V$ is a positive invertible operator. Then $A=U V^{1 / 2} \in G L O D\left(H^{+}\right)$by Proposition 2 and Lemma 7 , so $A$ is normal. This yields $V=U^{*} V U$ and $[U, V]=0$. Now it is routine to check that $U$ commutes with $Z_{H^{+}}$. (Note that facial homogeneity is useless.)

(b) $\Rightarrow$ (a): Let $U|A|$ be the polar decomposition of $A \in G L O D\left(H^{+}\right)$. Since $|A| \in Z_{H^{+}}$and $U \in U\left(H^{+}\right)$[8, II.3.2], $U$ commutes with $|A|$ by hypothesis thus $A$ is normal.

(ii) Let $U \in U\left(H^{+}\right)$. By (i) it is equivalent to show that $U \in S\left(H^{+}\right)$. Let $P$ be a projection in $Z_{H^{+}}$. Then $P \in O D\left(H^{+}\right)$and $U P$ is paranormal by hypothesis so

$$
0 \leq P U^{*} P U P-2 \lambda P+\lambda^{2} \quad \text { for all } \lambda \in \mathbf{R}^{+}
$$


Remark that $U^{*} P U \in Z_{H^{+}}$and $Q=U^{*} P U P \in Z_{H^{+}}$satisfies $Q \leq P$. Moreover, $Q-2 \lambda P+\lambda^{2} \geq 0 \forall \lambda \in \mathbf{R}^{+}$so $Q \geq P$ and $U P=P U P$. Exchanging the role of $U$ and $U^{*}$ we obtain $[U, P]=0$ and $U \in S\left(H^{+}\right)$by spectral theory.

Lemma 13 (see $[16,3.1,3.2])$. Let $H^{+}$be a self-dual cone.

(i) If any o.d. homomorphism is normal then $O D\left(H^{+}\right) \subset\left(Z_{H^{+}}\right)^{\prime}$ (commutant of $\left.\mathrm{Z}_{\mathrm{H}^{+}}\right)$and $U\left(\mathrm{H}^{+}\right)=S\left(\mathrm{H}^{+}\right)$.

(ii) The following conditions are equivalent:

(a) If $A \in O D\left(H^{+}\right)$then $A$ is normal.

(b) If $A \in O D\left(H^{+}\right)$and $A$ is a partial isometry then $A$ is normal.

Proof. (i) Let $A \in O D\left(H^{+}\right)$and $P$ be a projection in $Z_{H^{+}}$. Then $A$ and $A P \in$ $O D\left(H^{+}\right)$are normal. This implies $A^{*} P A=(P A)^{*} P A=P A(P A)^{*}=P A A^{*} P=$ $P A^{*} A P=A^{*} A P$ because $A^{*} A \in Z_{H^{+}}$. Hence, $(A P-P A)^{*}(A P-P A)=0$ and $A$ commutes with $Z_{H^{+}}$by spectral theory.

(ii) (b) $\Rightarrow$ (a): Let $U|A|$ be the polar decomposition of $A \in O D\left(H^{+}\right)$. Since $U$ is a partial isometry in $O D\left(H^{+}\right)$(Lemma 6), $U$ is normal by assumption. Let $P$ be a projection in $Z_{H^{+}}$. Then $P U$ is also a partial isometry in $O D\left(H^{+}\right)$. The proof of (i) gives that $U$ commutes with $Z_{H^{+}}$so $[U,|A|]=0$ by Proposition 2 and $A$ is normal.

We do not know how to characterize the self-dual cones enjoying the property that any o.d. homomorphism is normal. However in the finite-dimensional case the answer is the indecomposability of the cone:

Proposition 14 (see $[16,3.5])$. Let $\mathrm{H}^{+}$be a facially homogeneous self-dual cone in a finite-dimensional Hilbert space. Then the following conditions are equivalent:

(i) If $A \in O D\left(H^{+}\right)$then $A$ is normal,

(ii) $H^{+}$is indecomposable.

Proof. (i) $\Rightarrow$ (ii): Suppose $H^{+}$is decomposable. Then by [8, I.3.2] there exists a face $F \in \mathscr{F}\left(H^{+}\right)$such that $H^{+}=F \oplus F^{\perp}$ and $P_{F} \in Z_{H^{+}}$. Let $\xi \in F$ and $\eta \in F^{\perp}$ be two nonzero vectors such that $\langle\xi\rangle$ and $\langle\eta\rangle$ are extreme faces in $F$ and $F^{\perp}$. Then by [2] there exists $U \in U\left(H^{+}\right)$such that $U \xi=\eta$. Since $P_{F} U$ is in $O D\left(H^{+}\right)$, it is normal and $\left[P_{F}, U\right]=0$. This gives a contradiction with $\eta=P_{F^{\perp}} \eta=P_{F^{\perp}} U P_{F} \xi=P_{F^{\perp}} P_{F} U \xi=0$.

We conclude with some equivalence for the cones with a trace vector.

Proposition 15 (see $[16,3.6])$. Let $\mathrm{H}^{+}$be a facially homogeneous self-dual indecomposable cone and $\xi$ be a quasi-interior trace vector. The following conditions are equivalent:

(i) If $A \in O D\left(H^{+}\right)$and $A \xi=\xi$ then $A$ is normal.

(ii) If $A \in O D\left(H^{+}\right)$and $A \xi=\xi$ then $A \in G L O D\left(H^{+}\right)$. 
(iii) Every unital Jordan homomorphism of $\mathscr{M}$ is a Jordan isomorphism.

Proof. (i) $\Leftrightarrow$ (ii): Let $A \in O D\left(H^{+}\right)$. Since $H^{+}$is indecomposable, $A \in$ $G L\left(H^{+}\right)$if and only if $A$ is normal. This follows from the fact that its polar decomposition is $\lambda U$ where $\lambda \in \mathbf{R}^{+}$and $U \in U\left(H^{+}\right)$.

(ii) $\Leftrightarrow$ (iii): The map: $\delta \in \mathscr{M} \rightarrow \delta \xi \in H$ is an order isomorphism from $\mathscr{M}$ onto $\langle\xi\rangle-\langle\xi\rangle$ [8, IV.2.4]. On another hand $\alpha$ is a unital Jordan homomorphism from $\mathscr{M}$ into $\mathscr{M}$ if and only if $\alpha(|\delta|)=|\alpha(\delta)|$ ([6] with same proofs).

Moreover, if $\delta=\delta^{+}-\delta^{-}$is the Jordan decomposition of $\delta$ in $\mathscr{M}[8$, III.2.3] then

$$
\begin{aligned}
\left\langle\delta^{+} \xi, \delta^{-} \xi\right\rangle & =\left\langle\delta^{-} \delta^{+} \xi, \xi\right\rangle \\
& =\left\langle\delta^{+} \circ \delta^{-} \xi, \xi\right\rangle \quad[8, \text { IV.2.1] } \\
& =0
\end{aligned}
$$

and $(\delta \xi)^{ \pm}=\delta^{ \pm} \xi$ thus $|\delta \xi|=|\delta| \xi$ where $|\delta|=\delta^{+}+\delta^{-}$

So if $A \in O D\left(H^{+}\right)$and $A \xi=\xi$, we have for $\delta$ in

$$
-\|\delta\| \xi=-\|\delta\| A \xi \leq A \delta \xi \leq\|\delta\| A \xi=\|\delta\| \xi
$$

and there exists $\alpha(\delta) \in \mathscr{M}$ solution of the equation $A \delta \xi=\alpha(\delta) \xi$. Clearly $\alpha$ is a unital Jordan homomorphism and every such $\alpha$ defines, via the same equation, an element $A \in O D\left(H^{+}\right)$leaving $\xi$ invariant.

\section{SEMIGROUPS OF o.d. HOMOMORPHISMS}

The following has been proven in [5] in the special case where $H^{+}$is the natural cone associated to a $\sigma$-finite von Neumann algebra. Using Proposition 2 one can check that the proofs can be extended for the following:

Proposition 16. Let $\mathrm{H}^{+}$be a self-dual cone.

(i) Let $A(t)$ be a $C_{0}$-semigroup on $H$ with generator $G$. The following conditions are equivalent:

(a) $A(t) \in O D\left(H^{+}\right)$for all $t$.

(b) If $\xi \in D(G)$ then $|\xi| \in D(G)$ and the Kato equality is satisfied

$$
G|\xi|=\lim _{t \downarrow 0} \frac{|\xi+t G \xi|-|\xi|}{t}
$$

(ii) Let $A(t)$ be a $C_{0}$-semigroup in $L\left(H^{+}\right)$and $G$ its generator. The following conditions are equivalent:

(a) $A(t) \in O D\left(H^{+}\right)$is normal for all $t$.

(b) $G$ is normal and the real part of its spectrum is bounded from above. Moreover $G+G^{*}$ is affiliated with $Z_{H^{+}}$.

(iii) Let $U(t)$ be a $C_{0}$-semigroup of unitary operators with generator $G$. The following conditions are equivalent:

(a) $U(t) \in U\left(H^{+}\right)$for all $t$.

(b) If $\xi \in D(G)$ then $|\xi| \in D(G)$ and if $\xi \in D(G) \cap H^{+}$and $\eta \in H^{+}$ are orthogonal then $\langle G \xi, \eta\rangle=0$. 


\section{EXTREME MAPS IN $L\left(H^{+}\right)$}

In this section we extend all the results of [4] concerning the extreme order preserving maps. In fact, the o.d. homomorphisms are extreme in $L\left(H^{+}\right)$.

By $O D\left(H^{+}, \xi, \eta\right)$ we denote the subset of $O D\left(H^{+}\right)$applying $\xi$ onto $\eta$ and similarly $L\left(H^{+}, \xi, \eta\right)=\left\{A \in L\left(H^{+}\right) \mid A \xi=\eta\right\}$. Ext(.) means the extreme points.

Lemma 17. Let $H^{+}$be a self-dual cone and $\xi$ be a separating vector for $Z_{H^{+}}$. Then

$$
O D\left(H^{+}, \xi, \eta\right) \subset \operatorname{Ext}\left(L\left(H^{+}, \xi, \eta\right)\right) \quad \text { for any } \eta \in H .
$$

Proof. Let $A=\lambda A_{1}+(1-\lambda) A_{2}$ be a convex decomposition of $A \in O D\left(H^{+}, \xi, \eta\right)$ in $L\left(H^{+}, \xi, \eta\right)$ with $\lambda \in[0,1]$. Since $A-\lambda A_{1} \in L\left(H^{+}\right)$, the self-duality of $H^{+}$implies for $\eta, \rho \in H^{+}$

$$
0 \leq\left\langle\lambda A_{1} \eta, \lambda A_{1} \rho\right\rangle \leq\langle A \eta, A \rho\rangle .
$$

The right-hand side is zero if $\eta$ and $\rho$ are orthogonal. This implies that $A_{i}$ is in $O D\left(H^{+}\right)$and

$$
\lambda(1-\lambda)\left(A_{1}^{*} A_{2}+A_{2}^{*} A_{1}\right)=A^{*} A-\lambda^{2} A_{1}^{*} A_{1}-(1-\lambda)^{2} A_{2}^{*} A_{2}
$$

is in $Z_{H^{+}}$. Since $A_{1}^{*} A_{2} \xi+A_{2}^{*} A_{1} \xi=A_{1}^{*} A_{1} \xi+A_{2}^{*} A_{2} \xi$ by hypothesis, $A_{1}^{*} A_{2}+$ $A_{2}^{*} A_{1}=A_{1}^{*} A_{1}+A_{2}^{*} A_{2}$. Thus $\left(A_{1}-A_{2}\right)^{*}\left(A_{1}-A_{2}\right)=0$ and $A_{1}=A_{2}=A$.

The above inclusion is strict in general as shown by the following:

Theorem 18 (see $[4 ; 13,6.2])$. Let $H^{+}$be a facially homogeneous self-dual cone. Then the following conditions are equivalent:

(i) $O D\left(H^{+}, \xi, \eta\right)=\operatorname{Ext}\left(L\left(H^{+}, \xi, \eta\right)\right)$ for all quasi-interior points $\xi, \eta \in$ $H^{+}$.

(ii) $H$ is a Banach lattice.

(iii) $D\left(H^{+}\right)$is the Hermitian part of an abelian von Neumann algebra.

(iv) $O D\left(H^{+}, \xi, \eta\right)^{+}$is not empty for each $\xi, \eta$ in $H^{+}$and $\eta \leq \xi$.

(v) Let $G$ be the generator of a $C_{0}$-semigroup of o.d. homomorphisms then $G \xi \in P_{\langle\xi\rangle} H$ for all $\xi \in H^{+} \cap D(G)$.

Proof. Let $\left(\mathscr{M}^{+}\right)^{-1}$ be the set of invertible positive elements of $\mathscr{M}$. Let $\xi$ be a quasi-interior point in $H^{+}$and $\delta \in\left(\mathscr{M}^{+}\right)^{-1}$.

First we show that $P(\delta) \in \operatorname{Ext}\left(L\left(H^{+}, \xi, P(\delta) \xi\right)\right)$ where $P(\delta)=2 \delta^{2}-\delta \circ \delta$ [8, III.4]:

$P(\delta)$ is a self-adjoint invertible element belonging to $L\left(H^{+}\right)$as its inverse [8, III.4.8]. Thus $P(\delta) \xi$ is quasi-interior in $H^{+}$because if $\rho \in H^{+}$satisfies $\langle\rho, P(\delta) \xi\rangle=0$ then $P(\delta) \rho \in\langle\xi\rangle^{\perp}=\{0\}$ so $\rho=0$ and $\langle P(\delta) \xi\rangle^{\perp}=\{0\}$ [8, I.1.6].

Let $P(\delta)=\lambda A+(1-\lambda) B$ be a convex decomposition of $P(\delta)$ in

$$
L\left(H^{+}, \xi, P(\delta) \xi\right) \quad \text { where } \lambda \in[0,1] .
$$


Thus $\mathbf{1}=\lambda P(\delta)^{-1} A+(1-\lambda) P(\delta)^{-1} B \in L\left(H^{+}\right)$. Since $P(\delta)^{-1} A, P(\delta)^{-1} B$ and 1 are in $L\left(H^{+}, \xi, \xi\right)$ and $\mathbf{1} \in O D\left(H^{+}, \xi, \xi\right)$, the previous lemma implies $A=P(\delta)$ because $\xi$ is separating for $\mathscr{M}\left[8\right.$, II.1.5] and $\mathscr{M} \supset Z_{H^{+}}[8$, I.2.7], so the claim is proved.

(i) $\Rightarrow$ (ii): Let $\delta \in\left(\mathscr{M}^{+}\right)^{-1}$. Then by hypothesis

$$
P(\delta) \in \operatorname{Ext}\left(L\left(H^{+}, \xi, P(\delta) \xi\right)\right) \subset O D\left(H^{+}, \xi, P(\delta) \xi\right) .
$$

Applying this argument to $\delta^{01 / 2}$ (the square root in $\mathscr{M}$ ) we get

$$
P(\delta)=P\left(\delta^{\circ 1 / 2} \circ \delta^{\circ 1 / 2}\right)=P\left(\delta^{\circ 1 / 2}\right)^{2} \in O D\left(H^{+}\right) \quad[8, \text { III.4.4] }
$$

So by continuity $P(\delta) \in O D\left(H^{+}\right)$for all $\delta$ in $\mathscr{M}^{+}$. Remark 9 implies that $H$ is a Banach lattice.

(ii) $\Rightarrow$ (i): If $H$ is a Banach lattice then orthogonality and disjointness coincide in $H^{+}$. Thus the implication is a consequence of a Phelps-Ellis-Nagel's theorem [10, III.9.2]

(ii) $\Leftrightarrow$ (iii): The Jordan center of $\mathscr{M}$ coincides with $Z_{H^{+}}[8$, III.3.6] thus the equivalence follows from [8, I.3.2].

The implication (ii) $\Rightarrow(v)$ is proved in [9].

(ii) $\Rightarrow$ (iv): Every vector being a trace vector [8, IV.2.1], $\eta=\delta \xi[8$, IV.2.3] with $\delta_{2} \in \mathscr{M}^{+}$. Since $\mathscr{M}^{+}=\left(Z_{H^{+}}\right)^{+} \subset O D\left(H^{+}\right)$(Proposition 2), $O D\left(H^{+}, \xi, \eta\right)^{+}$ $\neq \varnothing$.

(iv) $\Rightarrow$ (ii): This is nothing but the Radon-Nikodym property [11, Proposition 1].

(v) $\Rightarrow$ (iii): Let $\delta=-\delta^{*} \in D\left(H^{+}\right)$. Then $e^{\delta} \in U\left(H^{+}\right) \subset O D\left(H^{+}\right)$and $\delta \xi \in P_{\langle\xi\rangle} H \forall \xi \in H^{+} \cap D(\delta)=H^{+}$by hypothesis. Thus when $\xi \in F^{\perp \perp}=P_{F} H^{+}$, $P_{\langle\xi\rangle} H \subset P_{F} H$ and $\delta \xi \in P_{F} H$. So $\left[\delta, P_{F}\right]=0 \quad \forall F \in \mathscr{F}\left(H^{+}\right)$and $\delta \in Z_{H^{+}}$by [8, I.2.7]. Consequently $\delta$ must be self-adjoint [8, I.2.7] so $\delta=0$. In particular if $\delta_{1}, \delta_{2} \in \mathscr{M},\left[\delta_{1}, \delta_{2}\right]=0$ and $\mathscr{M}$ coincides with its Jordan center thus with $Z_{\mathrm{H}^{+}}$which is the self-adjoint part of an abelian von Neumann algebra.

Remark 19. We proved that if $\delta \in\left(\mathscr{M}^{+}\right)^{-1}$ then $P(\delta) \in \operatorname{Ext}\left(L\left(H^{+}, \xi, P(\delta) \xi\right)\right)$ $\forall \xi \in H^{+}$. More generally if $A \in G L\left(H^{+}\right)$then $A \in \operatorname{Ext}\left(\left(L\left(H^{+}\right), \xi, A \xi\right)\right)$. This means that $U \in \operatorname{Ext}\left(L\left(H^{+}, \xi, \xi\right)\right)$ for all quasi-interior trace vectors $\xi$ of $H^{+}$ when $U \in S\left(H^{+}\right)$.

This motivates the following:

Lemma 20. Let $\mathrm{H}^{+}$be a facially homogeneous self-dual cone:

(i) Let $\xi$ be a quasi-interior trace vector in $\mathrm{H}^{+}$and assume $\mathrm{H}^{+}$is indecomposable. Then $\operatorname{Ext}\left(L\left(H^{+}, \xi, \xi\right)\right) \cap G L\left(H^{+}\right)=S\left(H^{+}\right)$.

(ii) $\operatorname{Ext}\left(L\left(H^{+}, \xi, \xi\right)\right) \cap G L\left(H^{+}\right)^{+}=\{\mathbf{1}\}$ for any quasi-interior $\xi$ in $H^{+}$.

Proof. (i) If $A \in \operatorname{Ext}\left(L\left(H^{+}, \xi, \xi\right)\right) \cap G L\left(H^{+}\right)$then $A \in S\left(H^{+}\right)$[8, IV.2.6] because $Z_{H^{+}} \approx \mathbf{R}$. The converse follows from the previous remark. 
(ii) Let $A \in \operatorname{Ext}\left(L\left(H^{+}, \xi, \xi\right)\right) \cap G L\left(H^{+}\right)$such that $A$ is positive as operator. Then $A=e^{\delta}$ for some $\delta \in \mathscr{M}$ [8, II.2.3] and $\xi=e^{\delta} \xi$ implies $\delta \xi=0$ and $A=1$. The converse follows from Lemma 17 since $1 \in O D\left(H^{+}\right)$.

We finish with the following problem that we have only scratched:

Characterize the set $L\left(H^{+}, \xi, \eta\right)$.

\section{REFERENCES}

1. H. Araki, An application of Dye's theorem on projection lattices to orthogonally decomposable isomorphisms, Pacific J. Math. 137 (1989), 1-13.

2. J. Bellissard, B. Iochum and R. Lima, Homogeneous and facially homogeneous selfdual cone, Linear Algebra Appl. 19 (1978), 1-16.

3. A. Connes, Charactérisation des espaces vectoriels ordonnés sous-jacents aux algèbres de von Neumann, Ann. Inst. Fourier (Grenoble) 24 (1974), 121-155.

4. T. B. Dang, Extremal maps of a Hilbert space equipped with a natural cone, Math. Z. 194 (1987), 95-97.

5. T. B. Dang and S. Yamamuro On homomorphisms of an orthogonally decomposable Hilbert space, J. Funct. Anal. 68 (1986), 366-373.

6. R. V. Kadison, A generalized Schwarz inequality and algebraic invariant for operator algebras, Ann. Math. 56 (1952), 494-503.

7. F. Hiai and S. Yamamuro, On homomorphisms of an orthogonally decomposable Hilbert space. III, J. Austral. Math. Soc. (to appear).

8. B. Iochum, Cônes autopolaires et algèbres de Jordan, Lecture Notes in Math., vol 1049, Springer-Verlag, 1984.

9. R. Nagel and H. Uhlig, An abstract Kato inequality for generators of positive semi-groups on Banach lattices, J. Operator Theory 6 (1981), 113-123.

10. H. H. Schaefer, Banach lattices and positive operators, Springer-Verlag, New York, 1974.

11. L. Schmitt, A remark on the Radon-Nikodym properties of ordered Hilbert spaces, Proc. Amer. Math. Soc. 105 (1989), 938-940.

12. S. Yamamuro, On orthogonally decomposable ordered Banach spaces, Bull. Austral. Math. Soc. 30 (1984), 357-380.

13. __ Absolute values in orthogonally decomposable spaces, Bull. Austral. Math. Soc. 31 (1985), 215-233.

14. __ Symmetry groups on ordered Banach spaces, Bull. Austral. Math. Soc. 33 (1986), 177-187.

15. __ On homomorphisms of an orthogonally decomposable Hilbert space. II, J. Austral. Math. Soc. 43 (1987).

16. On homomorphisms of an orthogonally decomposable Hilbert space. III, J. Austral. Math. Soc. 45 (1988).

17. _ On homomorphisms of an orthogonally decomposable Hilbert space. IV, C. R. Acad. Sci. Paris 305 (1987), 179-182.

18. _ On homomorphisms of an orthogonally decomposable Hilbert space. V, Research reports, No. 39, Australian National University, 1986.

19. _ Homomorphisms of an orthogonally decomposable Hilbert space, Research reports, No. 13, Australian National University, 1988.

Department of Mathematics, Université de Provence Marseille, Case 907, CPT, F 13288 Marseille Cedex 9, France 